\title{
ESTUDO DE AMOSTRAGEM EM EXPERIMENTOS COM HERBICIDAS
}

\section{RESUMO}

Tendo como um dos objetivos o estudo de amostrage $m$ foi instalado no dia 15 de março de 1981, um experimento no Centro Experimental de Campinas. O Delineamento Experimental utilizado foi em Blocos ao Acaso com três repetições, tendo sido testados nove diferentes herbicidas e mais dois tratamentos testemunha, com e sem cobertura com palha de capim-arroz.

Os canteiros, ou unidades experimentais, eram de dimensões $1,00 \mathrm{Om} \times 2, \mathrm{OOm}$ e em todos eles foram semeados alho (Allium sativum $\mathrm{L}$.) em linhas, espaçadas $0,25 \mathrm{~m}$ entre si, perfazendo quatro linhas por canteiro.

Para fins de avaliação dos efeitos de herbicidas cada canteiro foi dividido em três partes no sentido da linha e em 11 partes no sentido transversal, perfazendo um total de 33 pequenas áreas de dimensões $0,15 \mathrm{~m}$ x $0,30 \mathrm{~m}$ que constituiram as unidades básicas para fins de amostragem. Posteriormente, dentro de cada linha os pontos amostrais foram agrupados dois a dois, perfazendo ao todo 15 pontos de dimensões $0,30 \mathrm{~m} \times 0,30 \mathrm{~m}$, os quais serviram de base para compor as quatro áreas de amostragem indicadas a seguir:

$\mathrm{X}$ - uma área amostral de dimensões 9,30 $\mathrm{m} \mathrm{x}$ $0,30 \mathrm{~m}$ centrada no terço médio do canteiro;

X2 - uma área amostral centrada no terço médio do canteiro de dimensões $0,30 \mathrm{~m} \times 0,90 \mathrm{~m}$

(X está contido em X2);

X3 - uma área amostral centrada no terço mé dio do canteiro de dimensões $0,30 \mathrm{~m} \times 1,50 \mathrm{~m}$

(X2 está contido em X3);

X4 - uma área amostral composto de 3 áreas de dimensões $0,30 \mathrm{~m} \times 0,30 \mathrm{~m}$ tomadas em diagonal, um em cada terço (X1 está contido em X4).

A aplicação da Função discriminante aos dados de peso de matéria seca permitir am tirar as seguintes conclusões:

a) para avaliação dos efeitos de hebicidas às plantas daninhas da classe dicotiledôneas as áreas amostrais devem ser centradas no terço médio e correspondente a $4,5 \%$ do canteiro;

\section{T. IGUE* R.T. PAVEZI ${ }^{* *} \&$ E.M. PAULO***}

* Pesquisador Científico, Seção de Técnica Experimental e Cálculo.

** Enge nheiro-Agrôno mo, Bols is ta do CNPq, Estagiária.

*** Pesquisador Científico, Centro Experimental Campinas, Instituto Agronômico, Caixa Postal 28, 13100 - Campnias - SP.

b) para as monocotiledôneas as áreas amostaris devem ser também centradas no terço médio do canteiro e correspondente a $22,5 \%$ do mesmo.

PALAVRAS CHAVE: Area de amostragem, herbicidas, experimentos, plantas daninhas.

\section{SUMMARY}

SAMPLING IN HERBICIDE EXPERIMENTS

An experiment testing 9 different herbicides applied for weed control in garlic crop (Allium sativum L.) was used in order to define the sampling technique.

The following four sampling methods were compared through the Discriminant Function:

1) X1 - sample of $0,30 \mathrm{~m} \times 0,30 \mathrm{~m}$ size located at the central part of the plot;

2) X2 - sample of $0,30 \mathrm{~m} \times 0,90 \mathrm{~m}$ locted at the central third part of the plot (X1 is included in $\mathrm{X} 2$ );

3) X3 - sample of 0,30 x 1,50m also locted at the central third part of the plot (X2 is included in $\mathrm{X} 3$ );

4) X4 - three samples of the same size of $X 1$ with diagonal disposition(X1 is included in

x4).

The following conclusions were obtained: 1) Sampling representing $4,5 \%$ of the plot area was enough for evaluating the herbicides efficiency on dicotyledonous weeds;

2) for the monocotiledon class the sampling area should be $22,5 \%$ of the plot.

KEYWO RDS: Sampling areas, herbicides, trials, weeds.

\section{INTRODUÇÃO}

As plantas daninhas concorrem com as culturas por água, luz, espaço e nutrientes, além de servirem de hospedeiras de pragas e agentes causadores de moléstias, influindo assim na produtividade. 
A escassez de mão de obra na zona rural tem resultado na utilização cada vez maior de herbicidas. A pesquisa, através da experimentação agronômica, define os herbicidas para as diversas culturas passiveis de uso pelos agricultores.

Um dos problemas com relação aos experimentos com herbicidas pode surgir por ocasião da coleta de dados, isto é, a avaliação da eficiência desses produtos sobre as plantas daninhas quando, via de regra, se executa a contagem, a pesagem e a classificação botânica das infestantes remanescentes. Esse trabalho bastante moroso é diminuido tomando-se amostras nas unidades experimentais pois, dependendo da cultura, elas podem ter grandes dimensões.

Alguns trabalhos têm sido feitos para definir o tamanho das amostras a ser utilizado na avaliação dos experimentos com herbicidas. Igue et al. (1) chegaram à conclusão de que uma área amostral correspondente a $5 \%$ da unidade experi mental seria o ideal para avaliar tanto as monocotiledôneas como as dicotiledôneas remanescentes após a aplicação do herbicida. Perecin et ai. (3), testando dois métodos de amostragem, chegaram à conclusão de que 20 amostras de $0,1 \mathrm{~m} 2$ é mais eficiente que 4 amostras de $0,5 \mathrm{~m} 2$, correspondente a $10 \%$ da unidade expe rimental. Fazendo variar o número de repetições e o número de amostras por parcela esses mesmos autores concluiram que a estabilidade da variân cia média se consegue para amostras correspondentes a no máximo $3 \%$ e $5 \%$ da área útil da unidade experimental, respectivamente para dicotiledôneas e monocotiledôneas.

O objetivo do presente trabalho é definir o tamanho da amostra e alocação dos pontos amostrais nos canteiros para avaliação da eficiência dos herbicidas através dos dados de peso das plantas daninhas remanescentes pertencentes às classes das monocotiledôneas e dicotiledôneas.

\section{MATERIAIS E MÉTODOS}

O experimento com a cultura de alho (Allium sativum L.), variedade Lavinia, onde foram testados 9 tratamentos com herbicidas, além de dois tratamentos testemunhas, com e sem cobertura com palha seca de capmi-arroz, foi instalado no dia 14 de março de 1981, no Centro Experimental de Campinas.

Procurou-se uma variação na população de plantas daninhas no experimento através da aplciação de herbicidas, que foram os tratamentos, os quais são mostrados no Quadro 1, com suas respectivas doses utilizadas.

Quadro 1. Herbicidas e as respectivas doses utilizadas na cultura do alho.

Nome Comum

1. TRIFLURALIN

2. EPTC

3. OXADIAZON

4. LINURON

5. ALACHLOR

6. BUTACHLOR

7. MCPB

8. PENDIMETHALIN

9. DIPHENAMID
Nome Químico

$\alpha, \alpha, \alpha$-trifluoro-2,6-dinitro - N, N-dipropil-p-toluidine

S-etil diplopiltiocarbamato

2-tret-butil-4-(2,4 dicloro-5-isopropoxifenil)-2-1,3,4, -oxidiazolin5 -one

3-(3,4-diclorofenil)-1-metoxil-1-metil-ureia

2-cloro-2',6'-dietil-N-(metoximetil) acetanilida

2-cloro-2',6'-dietil-N-(butoximetil) acetanilida

$40 \%$ do ácido 2-metil-4-clorofenoxibutilico (MCPB); sob a

forma de sal sódico

$\mathrm{N}$-(1-etilpropil)-3,4-dimetil-2,6-dinitro benzonamina

$\mathrm{N}, \mathrm{N}$-dimetil-2,2-difenilacetamida

kg i.a./ha
0,80
4,33

0,88
1,25
1,94
2,65
1,60
1,75
5,00

As unidades experimentais, ou parcelas, eram de dimensōes $4,0 \mathrm{~m} \times 1,0 \mathrm{~m}$, sendo $2,0 \mathrm{~m}$ cobertos com palha de capim-ar- do foi o de Blocos ao Acaso com três repetiçōes dos onze tratamentos estudados. 
roz e $2,0 \mathrm{~m}$ sem cobertura. Em cada parcela foi semeada quatro linhas ou fileiras de alho, sendo $0,25 \mathrm{~m}$ o espaçamento entre as linhas e de $0,15 \mathrm{~m}$ entre as plantas dentro da linha.

A aplicação dos herbicidas foi feita com um pulverizador de pressão variável de dois litros de capacidade munido com um bico 8002 de tal sorte que nunca houve interrupção da forma em leque do jato no momento de aspersão.

Os dados foram coletados 65 dias após a aplicação dos produtos, e o sistema utilizado foi a divisão da área útil da parcela em 33 retângulos de dimensões $0,15 \mathrm{~m} \times 0,30 \mathrm{~m}$, que posteriormente foram agrupados 2 a 2 para fins de cálculo, ficando três linhas com cinco quadrados de dimensões $0,30 \mathrm{~m}$ x $0,30 \mathrm{~m}(4,5 \%$ da área útil da parcela). Os três retângulos adjacentes à parte coberta da parcela foram desprezadas. A área utilizada para obtenção de dados foi aquela correspondente a parte sem cobertura com palha de capim-arroz.

As plantas daninhas foram contadas e acondicionadas em sacos de papel, sendo cada retângulo considerado uma porcentagem da área útil da parcela, 2,25\% ou $0,045 \mathrm{~m}^{2}$. A secagem das plantas foi feita em secadora de $60^{\circ} \mathrm{C}$.

O procedimento estatístico utilizado para definir o método de amostragem se apoiou nos resultados obtidos por Pavezi (2) em experimentos com herbicidas em cultura de cebola (Allium Cepa L.), onde, através de estudo de componentes de variância, segundo o modelo, Yijk $=\mu+B i+L j(i)+A k(i)(j)$, sendo $\mu$ a média geral, Bi efeito do i-ésimo Bloco, $\mathrm{Lj}$ (i) efeito da $\mathrm{j}$-ésima linha do i-ésimo Bloco, e Ak(i) (j) efeito da k-ésima amostra da j-ésima linha do i-ésimo bloco.
Os componentes de variância

$$
\sigma_{B}^{2}, \sigma_{L}^{2}, \sigma_{A}
$$

estimados mostraram que existe pouca variação entre as amostras tomadas nas diferentes linhas. Diante desse resultado concentrou-se o estudo de amostragem na linha central, isto é, no terço médio da parcela.

Através de uma função discriminante, conforme Tatsuoka (5), foram comparados os quatro métodos de amostragem. O processo de coleta de dados dentro de uma parcela ou canteiro é apresentado a seguir :

\begin{tabular}{|c|c|c|c|c|}
\hline 1 & 2 & 3 & 4 & 5 \\
\hline 6 & 7 & 8 & 9 & 10 \\
\hline 11 & 12 & 13 & 14 & 15 \\
\hline
\end{tabular}

As tomadas de amostras ou métodos de amostragem são representados pelos quadrados enumerados como se segue:

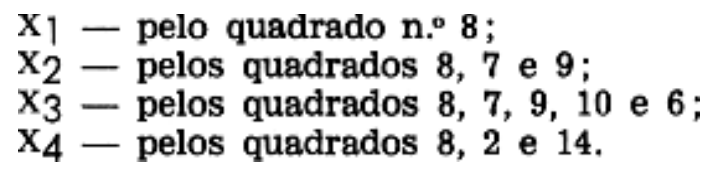

Para fins de análises estatísticas os dados de peso de matéria seca das plan tas daninhas tanto das monocotiledôneas como das dicotiledôneas foram transformados em $\log (\mathrm{X}+\mathrm{k})$, sendo $\mathrm{X}$ o peso de matéria seca obtido e $\mathrm{k}$ uma constante estimada segundo Prodan (4).

\section{RESULTADOS E DISCUSSÃO}

Para as plantas daninhas dicotiledôneas, as matrizes simétricas das somas dos quadrados e produtos cruzados: Total (T), Tratamentos (B), Blocos (C) e Erro (E) foram: 


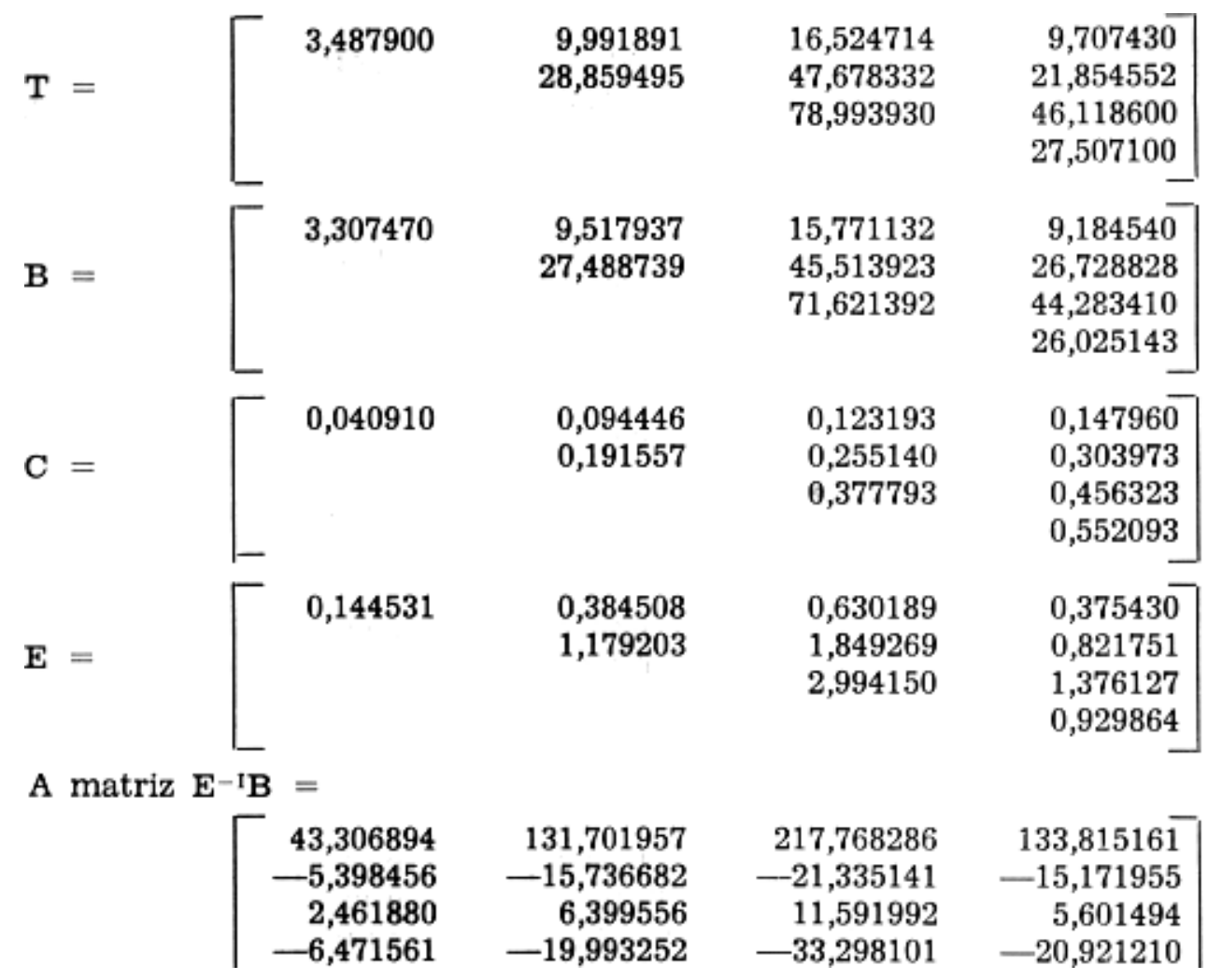

O polinômio característico obtido a partir da matriz $\mathrm{E}^{-1} \mathrm{~B}$ é :

$F(\lambda)=\lambda^{4}-18,24099329 \lambda^{3}-82,24607672 \lambda^{2}+73,56068 \lambda-17,724504$

As raizes caracteristicas ou autovalores (eigenvalues) são:

$$
\begin{aligned}
& \lambda_{1}=21,84942818 \\
& \lambda_{2}=0,938641823 \\
& \lambda_{3}=0,1987523 \\
& \lambda_{4}=-4,3483244
\end{aligned}
$$

Utilizando a maior raiz característica chega-se à seguinte função ajustada:

$$
\begin{aligned}
& \text { Y } 1=-0,371774 \mathrm{X} 1 \quad 0,148232 \mathrm{X} 2 \\
& -0,125780 \mathrm{X} 3 \quad 0,135714 \mathrm{X} 4
\end{aligned}
$$

Esta foi a única função que discriminou significativamente os tratamentos, e a variável $\mathrm{X} 1$ foi a que apresentou maior coeficiente, o que sugere que o método X1 de amostragem, isto é, um ponto amos- trai no centro da unidade experimental corres pondendo a $4,5 \%$ da parcela, é o mais indicado para fins de avaliação do efeito de herbicidas sobre as plantas daninhas dicotiledôneas na cultura de alho. Para as plantas daninhas da classe monocotiledônea as matrizes simétricas das somas dos Quadrados e produtos cruzados : Total (T ), Tratamentos (B), Blo$\cos (\mathrm{C})$ e Erro (E) foram: 


\begin{tabular}{|c|c|c|c|c|}
\hline $\mathrm{T}=$ & 0,278784 & $\begin{array}{l}0,708917 \\
2,177294\end{array}$ & $\begin{array}{l}0,912645 \\
2,980713 \\
4,572946\end{array}$ & $\begin{array}{l}0,410530 \\
1,211612 \\
1,917881 \\
0,969088\end{array}$ \\
\hline $\mathbf{B}=$ & 0,076292 & $\begin{array}{l}0,306217 \\
1,232223\end{array}$ & $\begin{array}{l}0,518737 \\
2,071055 \\
3,607426\end{array}$ & $\begin{array}{l}0,234856 \\
0,939681 \\
1,613707 \\
0,728387\end{array}$ \\
\hline $\mathrm{C}=$ & 0,044263 & $\begin{array}{l}0,068689 \\
0,113601\end{array}$ & $\begin{array}{r}0,068943 \\
0,106307 \\
0,107449\end{array}$ & $\begin{array}{l}0,055225 \\
0,071153 \\
0,087429 \\
0,099098\end{array}$ \\
\hline $\mathbf{E}=$ & 0,158229 & $\begin{array}{l}0,334011 \\
0,828470\end{array}$ & $\begin{array}{l}0,324965 \\
0,803351 \\
0,858071\end{array}$ & $\begin{array}{l}0,120449 \\
0,200778 \\
0,216745 \\
0,141603\end{array}$ \\
\hline
\end{tabular}

A matriz $\mathbf{E}^{-1} \mathbf{B}=$

$\left[\begin{array}{rrrr}-16,214180 & 4,209764 & 0,666670 & 8,461576 \\ -65,280741 & 17,153373 & \mathbf{2 , 4 7 4 9 3 6} & 34,054581 \\ -109,248502 & 27,088910 & 5,815541 & \mathbf{5 7 , 0 1 3 1 5 1} \\ -49,805238 & 12,669782 & 2,311946 & 26,005534\end{array}\right]$

O polinômio característico resultante da matriz $\mathbf{E}^{-1} \mathbf{B}$ é :

$$
F(\lambda)=\lambda^{4}-32,759668 \lambda^{3}+41,726361 \lambda^{2}-0,650821 \lambda+0,000485 \text {, }
$$

sendo as raizes características :

$$
\begin{aligned}
& \lambda_{1}=31,432851 \\
& \lambda_{2}=1,311034 \\
& \lambda_{3}=-0,014998 \\
& \lambda_{4}=0,000785
\end{aligned}
$$

O autovetor ajustado resultante do maior autovalor ou maior raiz característica foi :

$$
\begin{gathered}
\mathrm{Y} 1=0,046317 \mathrm{X} 1+0,424489 \mathrm{X} 2+ \\
0,740398 \mathrm{X} 3+0,135120 \mathrm{X} 4
\end{gathered}
$$

Esta é a única função que discrimina significativamente os tratamentos.

Observe-se que a variável $\mathrm{X} 3$ ou $\mathrm{o}$ método X3 de amostragem é o que apresenta o maior coeficiente, sugerindo ser este o método mais indicado para a ava- liação dos herbicidas sobre as monocotiladôneas na cultura do alho.

$\mathrm{O}$ método X3 corresponde à amostragem de $22,5^{\circ} \%$ o da parcela, sendo a localização das amostras no terço médio do canteiro.

Observe-se que tanto nas dicotiledôneas a faixa de amostragem foi definida como sendo o terço médio da parcela. A diferença observada no percentual do terço médio amostrado para mono e dicotiledôneas deveu-se à distribuição das 
plantas daninhas remanescentes. O que realmente verificou-se no campo, no momento da coleta de dados, foi as monocotiledôneas apresentando maior desuniformidade de distribuição do que as dicotiledôneas. Dessa forma pode-se concluir que quanto mais uniformemente se distribuem as plantas daninhas menor poderá ser a área amostrada no terço médio das parcelas.

\section{AGRADECIMENTOS}

Nossos agradecimentos ao Pesquisador Científico Reinaldo Forster, Chefe do Centro Experimental Campinas, do Instituto Agronômico, pelos incentivos e sugestões apresentadas no desenvolvimento do presente trabalho.

\section{LITERATURA GITADA}

1. Igue, T., R. Forster e R. Deuber. Amostragem mínima em contagem de ervas em experimentos com herbicidas. In: Seminário Brasileiro de Herbicidas e Ervas Daninhas, 10:, Santamaria, RS, 1974. p. 54-55. Resumos.

2. Pavezi, R.T. Amostragem em Experimentos com Herbicidas. IAC, Campinas, SP, 1981. 2. Relatório apresentado ao CNPq. 26p.

3. Perecin, D., R. Vitoria F** e L.R. Antiqueira. Amostragem para Avaliaçāo de Herbicidas em Cana-de-Açúcar. Cientifica, 4: 211-216, 1976.

4. Prodan, M. Forest Biometrics. Oxford, Inglaterra, Pergamon Press., 1968.

5. Tatsuoka, M.M. Multivariate Analysis. Tecniques for Educational and Psychological Research. New York, E.U.A., John Wiley \& Sons, Inc., 1971. 310 p. 